\title{
Review of: "Routes of transmission of SARS-CoV-2 and behaviours to block it: a summary"
}

\author{
Sharon $\operatorname{Cox}^{1}$ \\ 1 London South Bank University
}

Potential competing interests: The author(s) declared that no potential competing interests exist.

An insightful overview of the personal protective behaviours which play a role in the transmission of Covid-19. Population data is revealing that the pandemic is having a greater impact on society's most disadvantaged and vulnerable groups. As stated in this article, capability is essential. That is, while motivation remains important, central to protecting oneself and others is access to resources and knowledge. The figure provided in this article is a useful tool for community based stakeholders to use when working with groups disproportionately affected by the health and social burdens of the disease. For example, understanding where specific risks arise for individuals, i) who cannot work from home, ii) who cannot afford to not work, or take a reduction in pay, iii) without social networks for support, and iv) those who spend time in overcrowded conditions.

In developing this work, it is worth considering smokers from disadvantaged groups who are likely to engage in risky smoking practices. Risky smoking practices are defined here as those behaviours which increase the already high risk of smoking through the acquisition and use of cigarettes (e.g., sharing cigarette 'going twos', asking strangers for and smoking discarded cigarettes). Risky smoking practices are associated with the prevalence of respiratory infection. Using this article as a guide, I have outlined the main issues here in this presentation. *

*It is important to note, this is a separate issue to whether nicotine or smoking is protective. The point within this presentation is purely around routes of transmission. 